\title{
Superconducting Lead Granular Layer Prepared by Reducing in Hydrogen Lead of Germanate Glasses
}

\author{
J. Gackowska*, M. Gazda, K. Trzebiatowski and B. Kusz \\ Gdańsk University of Technology \\ Narutowicza 11/12, 80-952 Gdańsk, Poland
}

\begin{abstract}
The thin layer of $\mathrm{Pb}$ granules on the surface of the lead-germanate glass was prepared by thermal annealing in hydrogen. The structure and superconducting properties of lead layer depend on temperature and time of reduction. The influence of time and temperature of reduction on the properties of the reduced layer thickness has been studied.
\end{abstract}

PACS numbers: 61.43.Fs, 68.35.Bs, 68.37.Ps, 71.23.Cq

\section{Introduction}

Progress in nanotechnology opens many possibilities of practical glass applications. Those potential capabilities create necessity of fundamental properties studies. Thin films prepared by deposition onto cold substrates are interesting investigation object through that their sheet resistance $R_{\square}$ can be continuously varied by a slight thickness increment.

Another method of metal layer production is the reduction in hydrogen of the glass surface [1]. In this way layers of $\mathrm{Bi}$ on reduced bismuth-germanate and bismuth-silicate glasses may be obtained [2]. In the course of heat treatment in hydrogen $\mathrm{Bi}^{+3}$ ions are reduced into neutral atoms, which create the layer on the glass surface. A further reduction causes a rise in layer thickness. Such a procedure causes the surface conductivity increase by several orders. A similar process occurs in lead-germanate glasses. During the reduction process the layer of $\mathrm{Pb}$ grains is obtained on the surface. The grain size and layer thickness highly depend on the reduction time and temperature. This paper presents the analysis of the structure and electric properties of lead layer on the lead-germanate glasses. The authors found that despite various ways of formation and morphologic differences, results of $R_{\square}(T)$ are astonishingly consistent [3-5].

${ }^{*}$ corresponding author; e-mail: asia@mif.pg.gda.pl 


\section{Experiment}

The samples were prepared in traditional method $[2,6]$. The conductivity was measured in the temperature range from $4.8 \mathrm{~K}$ to $673 \mathrm{~K}$ in ambient atmosphere using two-terminal or four-terminal methods. The surface conductivity has been calculated from the equation $\sigma_{\square}=R^{-1} d / l$, where $R$ is the measured resistance, $d$ is the distance between electrodes and $l$ is the length of electrode. The surface of non-reduced and reduced samples was examined by X-ray diffraction using Philips X'Pert diffractometer system. AFM pictures were performed in air on microscope working with resolution of $5 \mathrm{~nm}$. We present results obtained for $\mathrm{Pb}_{0.3} \mathrm{Ge}_{0.7} \mathrm{O}_{1.7}$ samples reduced at $673 \mathrm{~K}$ for $2 \mathrm{~h}$ (Pb2), $3 \mathrm{~h}$ (Pb3), $15 \mathrm{~h}$ (Pb15), and $24 \mathrm{~h}$ (Pb24).

\section{Results}

The plot of surface conductivity versus reduction time of the $\mathrm{Pb}_{0.3} \mathrm{Ge}_{0.7} \mathrm{O}_{1.7}$ sample reduced at the temperature of $673 \mathrm{~K}$ is presented in Fig. 1. The curve presents a typical behavior for lead-germanate reduced glasses. First, after time of 1.3 hour a rapid increase (of a few orders of magnitude) in the surface conductivity appears. Next, the surface conductivity increases with a lower rate. This

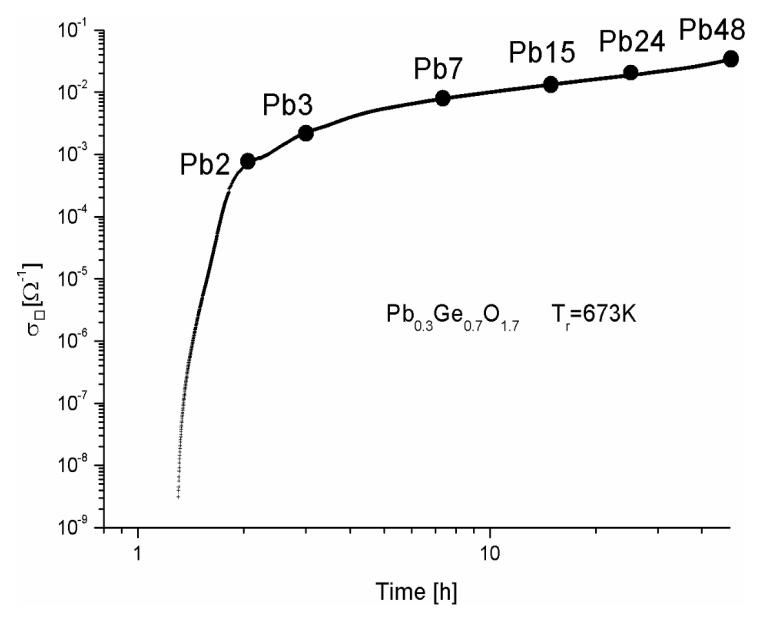

Fig. 1. The time dependence of conductivity $\mathrm{Pb}_{0.3} \mathrm{Ge}_{0.7} \mathrm{O}_{1.7}$ during reduction at the temperature of $673 \mathrm{~K}$.

measurement proves that the surface-conducting layer is thicker for a longer reduction time. The results of X-ray diffraction measurement of the studied samples are shown in Fig. 2. The plot of the non-reduced lead-germanate glass shows a typical of amorphous solids hallo pattern with no peaks. In the spectra of glasses annealed in hydrogen peaks characteristic of Fig. 2 crystalline lead may be seen. These peaks are larger in the glasses reduced for a longer time. The investigations also show that there is some quantity of Ge crystallites in the layer. It 


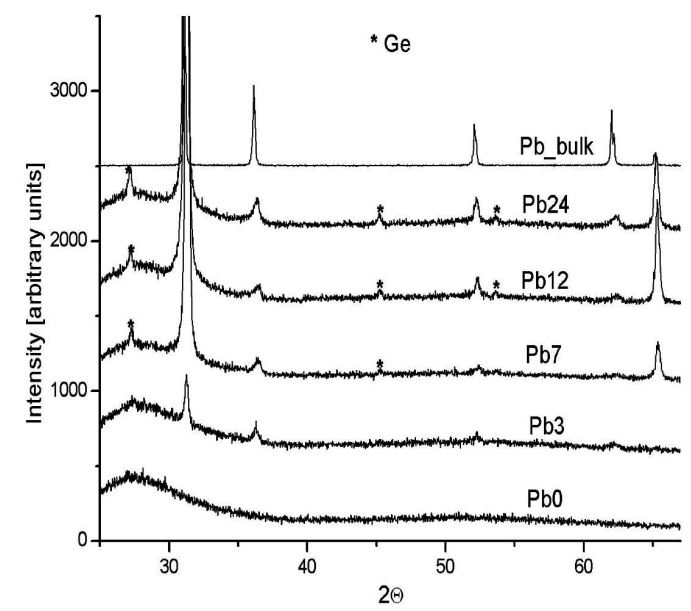

Fig. 2. X-ray diffraction spectra of samples $\mathrm{Pb0}-\mathrm{Pb} 24$ and bulk lead (Pb_bulk). Plots were shifted for better visibility. * denotes Ge spectra.

demonstrates that there proceeds a partial Ge reduction during heat treatment in hydrogen. Diffraction maxima of $\mathrm{Pb}$ in the thin layers are wider in comparison with the bulk lead. Estimated on the basis of the peak widening, the approximate diameter of the granules is about $50-100 \mathrm{~nm}$. Figure 3 presents representative results of surface morphology of the glass $(\mathrm{Pb0})$, the samples: after $2 \mathrm{~h}(\mathrm{~Pb} 2)$ and after 12 hours reduction in $400^{\circ} \mathrm{C}(\mathrm{Pb} 12)$. Glass surface is plain $( \pm 3 \mathrm{~nm})$ with small cracks made by polishing. In the case of two-hour reduction the $\mathrm{Pb}$ grains have the diameter of about $100 \mathrm{~nm}$ and are partially connected. In the regions

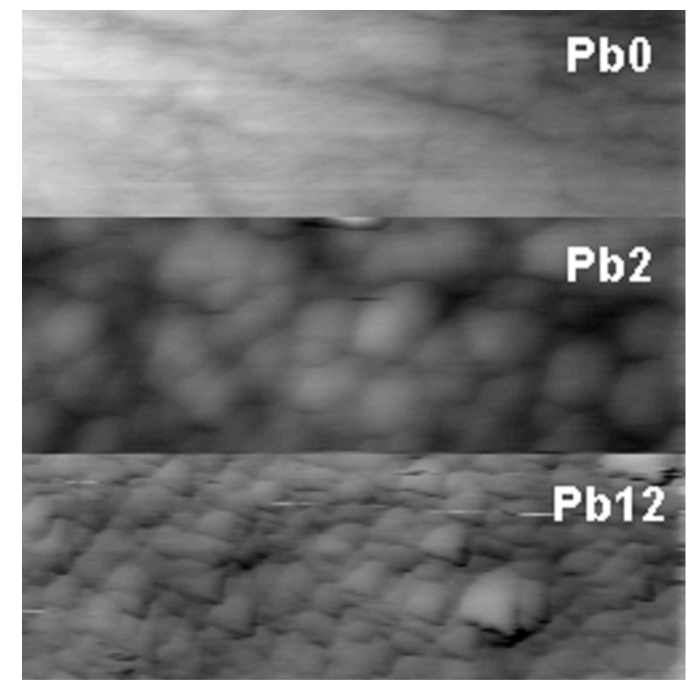

Fig. 3. Examples of AFM pictures of studied $\mathrm{Pb}_{0.3} \mathrm{Ge}_{0.7} \mathrm{O}_{1.7}$ glass samples. 
between the grains the glass surface is visible. On the surface of the Pb12 sample, with a more than $60 \mathrm{~nm}$ thick layer, homogeneous adhesion of the $\mathrm{Pb}$ crystallites layer is visible.

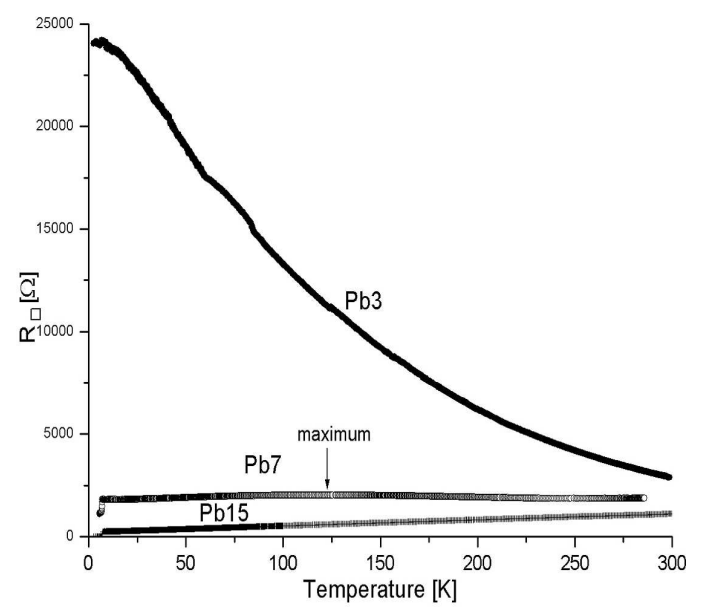

Fig. 4. The plots of surface resistivity versus temperature in $\mathrm{Pb}_{0.3} \mathrm{Ge}_{0.7} \mathrm{O}_{1.7}$ samples reduced for $3 \mathrm{~h}, 7 \mathrm{~h}$, and $15 \mathrm{~h}$ at the temperature of $673 \mathrm{~K}$.

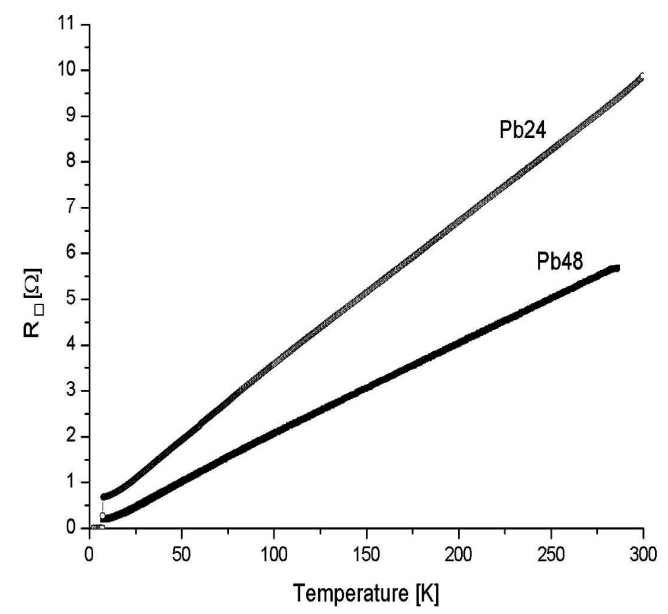

Fig. 5. The plots of surface resistivity versus temperature in $\mathrm{Pb}_{0.3} \mathrm{Ge}_{0.7} \mathrm{O}_{1.7}$ samples reduced for $24 \mathrm{~h}$ and $48 \mathrm{~h}$ at the temperature of $673 \mathrm{~K}$.

Figures 4 and 5 present the temperature dependence of the surface resistivity of the samples in the range from $5 \mathrm{~K}$ to $300 \mathrm{~K}$. It is seen that the surface resistivity of Pb3 (Fig. 4) decreases with temperature. In the case of Pb7 (Fig. 4) we observe the increase in $R_{\square}$ with temperature up to $125 \mathrm{~K}$ and above this temperature sheet resistivity decreases. Temperature dependence of resistivity of Pb15 (Fig. 4), Pb24 


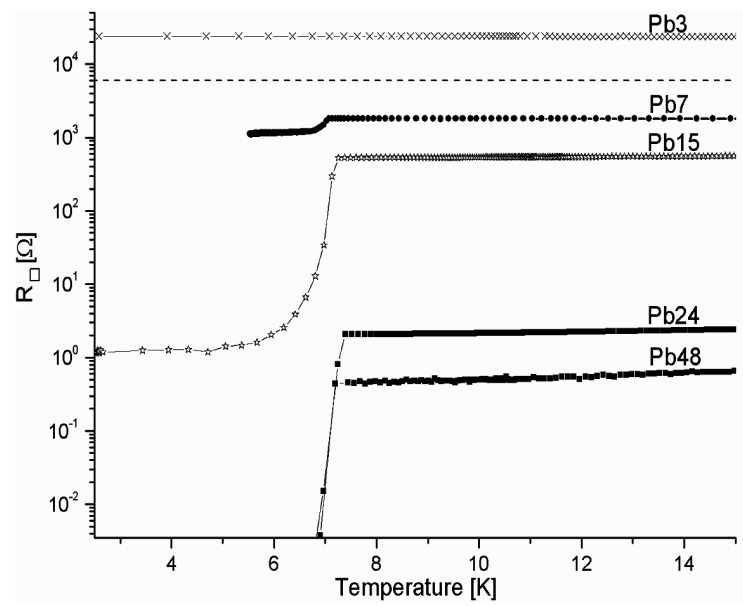

Fig. 6. The plots of surface resistivity versus temperature in samples $\mathrm{Pb} 3-\mathrm{Pb} 48$. The critical resistivity level of $R_{\text {crit }}=6.5 \mathrm{k} \Omega$ is marked by a dotted line.

and $\mathrm{Pb} 48$ (Fig. 5) has a typical metallic character. Figure 6 presents temperature dependence of $R_{\square}(T)$ obtained for all the studied samples in the range of $5-15 \mathrm{~K}$. The both metallic samples $(\mathrm{Pb} 24, \mathrm{~Pb} 48)$ transit into the superconducting state at $T_{\mathrm{C}}=7.2 \mathrm{~K}$. Plots received for $\mathrm{Pb} 7$ and $\mathrm{Pb} 15$ samples show a wide and uncompleted transition below a temperature of $T_{\mathrm{C}}=6.9 \mathrm{~K}$ and of $T_{\mathrm{C}}=7.1 \mathrm{~K}$, respectively.

\section{Discussion}

In the case of nano-metal layer the grain size can have an essential influence on the superconducting properties. Considerable changes of $T_{\mathrm{C}}$ as a function of the grain dimension of $\mathrm{Pb}$ were observed by Haviland et al. [7]. On the other hand, $\mathrm{Pb}$ layer produced in other conditions showed very small changes of $T_{\mathrm{C}}$ [8]. We can suppose that in the case of the layer, in which the grains are practically equal ( $D$ distribution is small), $T_{\mathrm{C}}$ will not change with layer thickness. Our results demonstrate that the layers produced using the reduction method consist of randomly distributed grains with a size of about 50-100 nm. Even in the first phase of reduction the size of grain is about $30-80 \mathrm{~nm}$. Transition insulator-metal during further reduction is caused by the formation of a larger number of grains. After achieving the percolation threshold the electrical conduction can occur either by electron tunneling or by direct contact of metal grains. The enlargement of layer thickness causes only a slight $T_{\mathrm{C}}$ increase (samples: from $\mathrm{Pb} 7$ to $\mathrm{Pb} 48$ ), because the grains have a similar size.

Our study shows that the reduction method of lead-germanate glass permits to obtain $\mathrm{Pb}$ layers of various electrical properties. The samples reduced for a short time $(\mathrm{Pb} 3)$ exhibit a semiconductor-like type of the temperature dependence of conductivity, while the samples reduced for longer times ( $\mathrm{Pb} 7-\mathrm{Pb} 24)$ have either uncompleted superconducting transition or are truly superconducting 
(Pb15, Pb24). The results presented in this paper agree qualitatively with those obtained by other authors [8], however the estimated $R$ value is about one order of magnitude smaller than the expected one. The reason of this difference is not known. We suppose that it is the influence of the fact that $\mathrm{Pb}$ layers contain a certain quantity of randomly distributed germanium phase. Its presence can disturb Josephson's junction continuous net creation process.

\section{Acknowledgments}

We would like to thank Prof. L. Murawski for his fruitful discussion and M. Kruk for preliminary measurements.

\section{References}

[1] B. Kusz, Solid State Commun. 125, 623 (2003).

[2] B. Kusz, K. Trzebiatowski, M. Gazda, L. Murawski, J. Non-Cryst. Solids 328, 137 (2003).

[3] R.A. Farrell, B. Mirhashem, Phys. Rev. B 37, 648 (1988).

[4] A.O. Caldeira, A.J. Leggett, Phys. Rev. Lett. 46, 211 (1981).

[5] A. Ambegaokar, U. Eckern, G. Schon, Phys. Rev. Lett. 48, 1745 (1982).

[6] J. Gackowska, M. Gazda, K. Trzebiatowski, B. Kusz, submitted to J. Non-Cryst. Solids.

[7] D.B. Haviland, Y. Liu, A.M. Goldman, Phys. Rev. Lett. 62, 2180 (1989).

[8] H.M. Jaeger, D.B. Haviland, B.G. Orr, A.M. Goldman, Phys. Rev. B 40, 182 (1989). 